\title{
3D RECONSTRUCTION FROM MULTI-VIEW MEDICAL X-RAY IMAGES - REVIEW AND EVALUATION OF EXISTING METHODS
}

\author{
S. Hosseinian*, H. Arefi \\ School of Surveying and GeoSpatial Engineering, University of Tehran, Tehran, \{saharhosseinian, hossein.arefi \}@ut.ac.ir
}

KEY WORDS: Stereoradiography, 3D Reconstruction, X-ray images, close range photogrammetry

\begin{abstract}
:
The 3D concept is extremely important in clinical studies of human body. Accurate 3D models of bony structures are currently required in clinical routine for diagnosis, patient follow-up, surgical planning, computer assisted surgery and biomechanical applications. However, 3D conventional medical imaging techniques such as computed tomography (CT) scan and magnetic resonance imaging (MRI) have serious limitations such as using in non-weight-bearing positions, costs and high radiation dose(for CT). Therefore, 3D reconstruction methods from biplanar X-ray images have been taken into consideration as reliable alternative methods in order to achieve accurate 3D models with low dose radiation in weight-bearing positions. Different methods have been offered for 3D reconstruction from X-ray images using photogrammetry which should be assessed. In this paper, after demonstrating the principles of 3D reconstruction from X-ray images, different existing methods of 3D reconstruction of bony structures from radiographs are classified and evaluated with various metrics and their advantages and disadvantages are mentioned. Finally, a comparison has been done on the presented methods with respect to several metrics such as accuracy, reconstruction time and their applications. With regards to the research, each method has several advantages and disadvantages which should be considered for a specific application.
\end{abstract}

\section{INTRODUCTION}

Nowadays, 3D reconstruction is an important issue in clinical studies for diagnosis and treatment of pathologies especially related to the bony structure of the human body. 3D models are used more extensively for operation planning and morphometric studies. Applying personalised 3D precise models by surgeons has great impact on the accuracy and reliability in orthopaedics. Medical imaging techniques make it possible to generate 3D models for assessment, quantification and prediction of deformation of bones. Conventional Medical techniques for 3D imaging are computed tomography (CT) scan and magnetic resonance imaging (MRI). CT-scan is an accurate common 3D imaging method but it induces a high radiation dose for the patient. In several diseases such as scoliosis which frequent scans are needed for clinical follow-up exams, this method would result in unacceptable radiation exposure risk. In addition, CT scan is expensive and performed with the patient in a lying position, where it changes the global shape of bone structures. This issue becomes more essential, particularly for diseases concerned to the spine and lower limb. Therefore, due to the mentioned limitations such as high irradiating dose and of the patient having to adopt a lying position, CT is not suitable for $3 \mathrm{D}$ evaluation of bone structure in several structure pathologies. On the other hand, Methods based on MRI does not have the problem of high irradiating dose but they are less accurate and more expensive than CT imaging and they are not appropriate for patients with ferromagnetic metallic implants or other ferromagnetic materials because of the potential risks associated with movement or dislodgment of these objects and also artifacts caused by metallic objects in MRI. Moreover, MRI is less applied for bone and it is more used for soft tissue. However, MRI-based methods can be adopted only in lying position, similarly. For these mentioned reasons, conventional 3D imaging techniques, MRI and CT scan, are inappropriate for obtaining 3D accurate models of bone structures. Hence, various researches have been done on introducing alternative methods which eliminate these limitations and at the same time provide accurate 3D information. Currently, using photogrammetry for creating 3D models from radiographic images has been taken into consideration extensively as a reliable alternative approach because of accuracy, low irradiating dose, and ability of being applied for standing position. Different methods have been offered for 3D reconstruction from X-ray images using photogrammetry concepts which should be assessed. In this paper, after demonstrating the principles of $3 \mathrm{D}$ reconstruction from X-ray images, different existing methods of $3 \mathrm{D}$ reconstruction of bony structure with low dose of irradiation are presented and classified. Then, their applications, advantages and disadvantages are evaluated. Finally, a comparison will be done on the limitations and accuracy of the results of implementation of these methods.

\section{PRINCIPLES OF 3D RECONSTRUION FROM RADIOGRAPHIC IMAGES}

For evaluation of 3D reconstruction methods, important aspects of $3 \mathrm{D}$ reconstruction from radiographic images should be considered which are as followed: (a) Acquisition of multi view $\mathrm{X}$-ray images, at least two images; These images can be captured by commercial standard radiographic devices or expensive specified solutions such as EOS imaging system which can acquire high quality calibrated stereo radiographic images simultaneously. Images can contain markers as benchmarks or not, with regards to the application and the required accuracy. Markers have different types such as those implanted into the bone by surgery which are rigid or those simply attached on the skin. Using markers brings us accuracy but, at the same time, it has some serious disadvantages such as inconveniency for the patients and costs which cannot be neglected. (b)image enhancement and restoration that is important for achieving acceptable results according to the nature of X-ray images especially when fluoroscopy images are

\footnotetext{
* Corresponding author
} 
used; X-ray images exhibits severe quantum noise due to limited X-ray photons involved. Because of the nature of signal-dependant quantum noise, Gaussian models are not suitable for modelling them. X-ray images are generally modelled by Poisson distribution which successfully models the photon counting statistics of imaging detectors. In addition, it should be considered that the quality of X-ray images is affected by the superimposition of bony structures(c) Calibration which should be done to generate 3D data from $2 \mathrm{~d}$ coordinates and it includes correction for image distortion, calculation of the focus position and calculation of the relative position and orientation of the images. Nowadays, calibrated images are available by means of some acquisition $\mathrm{x}$ ray systems. But in order to propose methods which can be used in clinical routines by standard radiographic systems, calibration scope should be evaluated precisely. (d)3D reconstruction methods with different levels of automation for data extraction and optimization and redefinition of generic models and obtaining specified 3D model which are considered in the evaluation of $3 \mathrm{D}$ reconstruction methods in the section 3 . In this paper, the focus is on the $3 \mathrm{D}$ reconstruction methods and further important assessments such as calibration are not evaluated here, because of the extent of the issues.

\section{EVALUATION OF STEREORADIOGRAPHIC 3D} RECONSTRUION METHODS

Stereoradiographic 3D reconstruction methods can be classified based on different measures such as level of automation, primitives and models which are used, objects and applications. Based on the level of automation, methods can be categorized in three groups which are manual, semi-automatic and automatic methods. This issue is mentioned for methods evaluated in the following. Moreover, there are different types of deformable models can be applied by reconstruction methods, such as geometric deformable models and statistical models. According to Markelj et al (2011), with respect to the nature of registration methods used for the reconstruction, they can be classified as feature based, intensity based and gradient based methods. However, most of the proposed methods are either feature-based or intensity based. Therefore, gradient based methods described in (Markelj et al, 2011) are not illustrated here. Feature-based methods use points, contours or surfaces which are extracted by segmentation procedures for 3D reconstruction. Therefore, their accuracy is dependent to the accuracy of segmentation. Also they are sensitive to the noise and shape of the features. Edge feature-based methods minimize the distance between object edges in the image and model silhouette, such as methods used in (Zheng et al., 2008; Fleute et al, 1999; Benameur et al., 2003, 2005). Intensity based methods rely on only information and intensity of the images. They optimize intensity similarity between the X-ray images and simulated X-ray projection images called digitally reconstructed radiographs (DRRs) which can be generated from CT-scan such as methods used in (Mahfouz et al 2006; Yao et al, 2003; Hurvitz et al, 2008; Tang et al, 2005). The success of Edge-feature based methods depends on the quality of the segmentation. However, they have lower computational complexity when compared to intensity based methods. In addition, according to the considered bony structure and the application, methods can be categorized into different groups such as methods for rib cage(Labele, 1995; Aubert et al, 2014), lower limb such as femur, tibia (Chaibi et al, 2010, 2011; Baka et al, 2o11; Quijano et al, 2013), pelvis (Gauvin et al, 1998; Mitton, 2006), vertebrae and spine (Benameur et al, 2003, 2005; Pomero et al, 2004; Kadoury et al, 2009, 2015; Humbert et al, 2009, Moura et al, 2011) and upper limb(Lebailly et al,
2012). Finally, by considering the mentioned aspects and various classifications have been already done by different authors, 3D reconstruction methods mainly can be classified as: (1) point-based, (2) contour-based, (3) statistical shape model based methods, (4) parametric and (5) hybrid methods. For each class, the algorithms, and the characteristics will be described.

\subsection{Point-based methods}

Point-based methods have been the first methods presented for 3D reconstruction from radiographic images. Methods of this class rely on identifying low-level primitives, points, and match them on multi-view radiographs. 3D reconstruction point-based techniques can be mainly divided into two main classes: StereoCorresponding Point based Techniques, and Non StereoCorresponding Point based Techniques which are explained as followed.

\subsubsection{Stereo-Corresponding Point Based Techniques}

These methods are implemented based on the stereo corresponding points manually identified in multi view radiographs by an expert operator. Therefore, they are called SCP methods. In these methods, the important issue is localization of at least six corresponding anatomical landmarks (Figure 1(a)). In preliminary presented point based methods, the first step was extracting corresponding points in two $\mathrm{x}$ ray images and the second step was 3D reconstruction with algorithms such as DLT that allows reconstruction only where there are SCPs. (Stockes et al., 1981; Pearcy, 1985; Dansereau et al, 1988; Andre et al., 1994) used SCP for 3D reconstruction. Stockes et al. (1981) and Pearcy (1985) generated 3D data from identifying 6-9 SCP, and then using DLT algorithm. But a reliable and precise detailed model was not obtained. Because of the weak results of methods considering only local information and with regards to universal patterns of anatomical structures, using of prior knowledge in 3D reconstruction from radiographs has been taken to consideration. In order to achieve more accurate results, for the first time, Aubin et al (1997) tried to build a morpho-realistic model by applying a generic model achieved from a 3D scanner and identifying SCP. The model was obtained by kriging the generic object using the six SCPs as control points. They used DLT algorithm for 3D reconstruction. The results were improved but there was still a need for more landmarks to obtain a better description of all the vertebrae. Aubin et al. (1997) and Gauvin et al. (1998) offered using more points for reaching more precise models. Gauvin et al, in (1998) applied this method for pelvis using more points (19 SCPs). Then they reconstructed the 3D points with DLT algorithm. Average deviation from direct measurements was (2SD) $4.8 \mathrm{~mm}$. Aubin et al, (1997) also applied this method for vertebra using more points (21 and 6 SCPs for each vertebra in each radiograph for comparison). For $21 \mathrm{SCPs}$, errors were $2.1 \pm 1.5 \mathrm{~mm}$ (mean \pm standard deviation) in comparison with 21 direct measurements. The results were improved but because of the time needed for identifying additional points, this increasing in SCPs becomes so time consuming and it was not suitable. In conclusion, the disadvantages of these methods are ambition in corresponding point identification, low reproducibility and time consuming. Besides, because of the limited number of corresponding anatomical landmarks identifiable on radiographs, these methods still have low accuracy.

\subsubsection{Non Stereo-Corresponding Point Based Techniques}


With regards to the limitations of SCP-based methods, non stereo-corresponding point based techniques (which can use points visible only on one radiograph) were proposed as an improvement of the previous techniques. These techniques are based on this principle that any non-stereo corresponding point (NSCP) belongs to a line joining the X-ray source and the projection of the point in one view. They enable the 3D reconstruction of additional landmarks that can be identified in only one of the radiographs (Figure 1(b)). These methods allow 3D reconstruction of both SCPs and NSCPs, to obtain a more refined and detailed 3D geometry by deforming generic models using an epipolar geometry. In these methods, an elastic object is deformed with respect to the SCPs and NSCPs on the radiographs. Mitton et al, (2000) was proposed an NSCP based method for $3 \mathrm{D}$ reconstruction of vertebrae. The calibration was done by means of the identified points. Subsequently, 3D reconstruction of SCPs (using the DLT algorithm) was performed. Then, the reconstruction of NSCPs and computing their 3D coordinates was done by definition of the line joining the source and the projection of an anatomical landmark in the radiograph, definition of a generic object, initialization of the points on their lines on the radiograph, optimization procedure to find the position of the points on their lines, and considering the shape similarity with the generic object. The final step was kriging of the generic object by means of the reconstructed points as control points. Mitton et al (2000) used this method to obtain the 3D geometry of upper cervical vertebrae. The reported precision determined with the point-to-surface distance between the reconstruction obtained with that technique and reference measurements was about $1 \mathrm{~mm}$, depending on the vertebrae studied. Because it used more information from the radiograph, the algorithm had been shown to provide a considerably more accurate $3 \mathrm{D}$ reconstruction, when compared to the DLT technique. Mitulescu et al (2001) applied this method for 3D reconstruction of dried lumbar vertebrae from biplane X-ray images. The study achieved reconstruction errors of $1.1 \pm 1.4 \mathrm{~mm}$ (mean \pm RMS). Mitulescu et al (2002) 3D reconstructed thoracic and lumbar vertebrae from biplane images using similar method. The 3D coordinates of the 25 anatomical landmarks were obtained as described: 6 SCPs were reconstructed using the DLT algorithm and 19 NSCPs were reconstructed using this algorithm. The validation of their technique on scoliotic patients was performed on 58 scoliotic vertebrae in 14 patients, by comparison with the CT scan. The results of this study show error of $1.5 \pm 2.0 \mathrm{~mm}$ (mean \pm RMS) with using NSCP method (using 6 SCPs plus 19 NSCPs).

a)

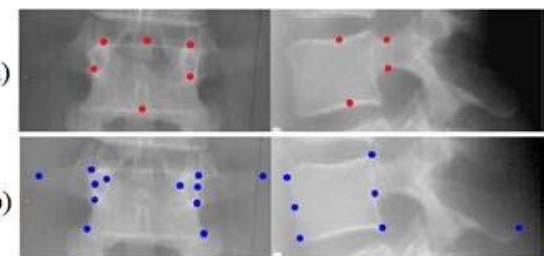

Figure 1. (a) Stereo corresponding points, (b) Non stereo corresponding points (A. Mitulescu et al, 2002)

Point-based methods are considerably dependent on the skill of the expert operator for identifying exact points. Therefore, they have low reproducibility. These methods need time of about 24 hours for reconstruction because of the manual identification.

\subsection{Contour-based Methods}

Beside the limitations of point-based methods such as time consuming and non-reproducibility, they cannot be used for bony structures with continuous shape, like knee joint, because of the lack of specific anatomical landmark points (S. Laporte et al, 2003). Laporte et al (2003) offered a contour based method for reconstruction of distal femur. They improved the stereo radiographic reconstruction methods especially for continues shape bone cases. They applied a deformable generic model using non stereo corresponding contours instead of points. The principle of the method is to associate identifiable 2D-contours from radiographs to 3D lines defined on the surface of a reference object and elastic 3D model deformation with regards to $2 \mathrm{D}$ contours available onto the different X-rays films. Therefore, this method is called the non stereo corresponding contour (NSCC) method. In NSCC algorithm, the preliminary step is calculation of an initial solution. The first step is definition of anatomical regions from the generic object; second step is manual $2 \mathrm{D}$ contours identification on the radiographs (figure $2 \mathrm{~A}$ ). Then, $2 \mathrm{D}$ contours from the $3 \mathrm{D}$ initial solution object should be generated for each radiographs. 3D contours of the initial object surface are projected onto their associated radiograph. Then, association between these 2 set points should be performed. This $2 \mathrm{D}$ association is based on point-to-point distances and contours derivations. This enables to obtain a correspondence between the $2 \mathrm{D}$ contours and the $3 \mathrm{D}$ contours. Next step is optimization of the initial solution. The final step is deformation of the optimized solution applying Kriging algorithm to the optimized solution (S. Laporte et al, 2003). Finally, the reconstructed object is obtained iterating final step as long as the distance between two set points is superior to a given precision value. They reported the mean error of $1.0 \pm 1.4 \mathrm{~mm}$ (mean error \pm RMS) and the maximum error of $5 \mathrm{~mm}$ for which were accurate and were close to those obtained generally using CT-scan. This method can be adapted to any structure as long as the requirements are fulfilled.
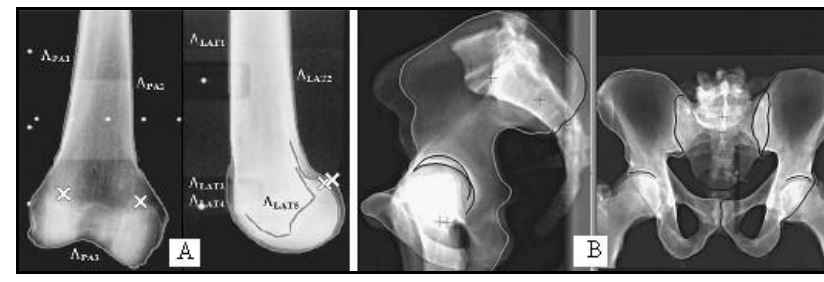

Figure 2. Identification of anatomical contours on the radiographs for: (A) distal femur (S. Laporte et al, 2003), (B) pelvis (S. Mitton et al, 2006)

Considering the complexity of the shape of pelvis, Mitton et al (2006) used a 3D reconstruction contour based method for pelvis and superior extremity of the femurs (figure $2 \mathrm{~B}$ ). They proposed a method progressively reconstructed a coarse initial model, an intermediate one and then the accurate personalized model. The reported results were close to CT-scan reconstructions with mean difference of $1.6 \mathrm{~mm}$. Other researchers also used contour-based methods, such as (Bauer, 2002) for femur and tibia and (Le Bras et al, 2004) for proximal femur. In contour based methods, higher level of geometric primitives are used, so the problem of finding corresponding points is avoided and it reduces the need for user intervention in comparison to point-based methods. Despite of the acceptable accuracy of contour-based methods, these methods still need considerable time for $3 \mathrm{D}$ reconstruction. The required time for these 3D reconstruction methods were reported about 15-35 minute for lower limbs which is time consuming for applying in clinical use. 


\subsection{Statistical Shape Model Based Methods}

Statistical shape model based methods are proposed as more automated reconstruction algorithms. They reduce user intervention with considering more information about pathologic objects, using statistical knowledge of possible deformations of the objects. Besides, they achieve accurate 3D reconstruction especially for objects such as vertebrae. These methods require a large learning database of shapes including normal and pathologic shapes of subjects. In these methods, a model containing information of the mean shape and its variations called statistical shape model (SSM) is applied. Principal Component Analysis (PCA) is used on the learning database in order to find model shape variations (e.g. figure 3). The deformation is done, until the deformed model's projection is coherent with the information of radiographs. In this class of methods, many researches have been done for 3D reconstruction of different objects such as vertebrae and spine (Fleute, 2001, Benameur et al, 2003, 2005), femur (Fleute et al, 1999; Baka et al, 2011) and pelvis (Yao et al, 2003, Sadowsky et al, 2006). In the following paragraphs, some of the important proposed statistical model based methods for 3D reconstruction from biplanar X-ray images are described.

Fleute et al (1999) proposed a statistical edge based method to build the 3D model of the distal femur. They applied point distribution models (PDM) in order to reconstruct a prior model. The statistical model was built based on PCA. Then, they deformed nonrigidly the 3D statistical model to the contours segmented on the $\mathrm{x}$ ray images (M. Fleute et al, 1999). The final RMS obtained between the deformed model and the shape was $0.99 \mathrm{~mm}$ with the computation time of less than one minute.

Benameur et al (2003) proposed a 3D reconstruction SSMbased method for each individual vertebra. They applied a database of normal and pathologic vertebrae. They used a prior global knowledge captured by a statistical deformable template integrating a set of admissible deformations, expressed by the first modes of variation in karhunen-loeve expansion of the pathological deformation (S. Benameur et al, 2003). They used canny edge detector to estimate the edge map and then the edge potential field on the two radiographs. Subsequently, they proposed a registration method consisted of fitting the deformable model with the segmented contours of the corresponding vertebra on two radiographs that leaded to an optimization problem which was by a gradient descent algorithm initialized by a rigid $3 \mathrm{D} / 2 \mathrm{D}$ registration. The final mean error was $0.71 \mathrm{~mm}$ for lumbar and $1.48 \mathrm{~mm}$ for thoracic vertebra.

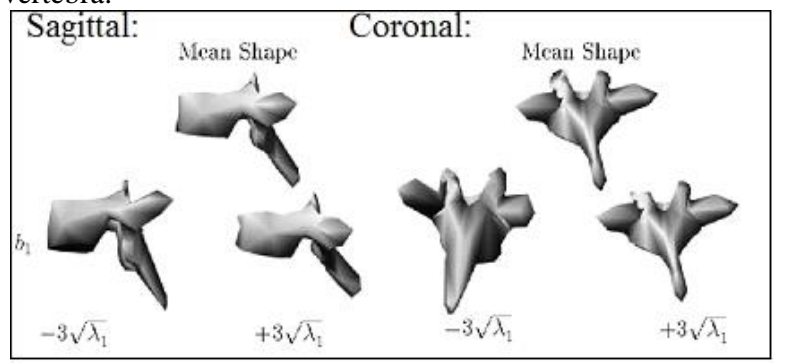

Figure 3. Fine prior model of each vertebra (S. Benameur et al, 2005). The mean shape and two deformed shapes for T8 vertebra from the sagittal and coronal views are shown.

To present a more automated algorithm, Benameur et al (2005) proposed a similar reconstruction method using hierarchical global a priori knowledge on the geometric structure of the whole spine in addition to each vertebra, for improving the 3D reconstruction. This method used two statistical models. The first statistical model is used to register the whole spine and determine an initial solution. Then, accurate 3-D reconstruction is performed for each vertebra by a second model on which nonlinear admissible global and local deformations are defined. Their coarse-to-fine 3D reconstruction procedure leads to two separate minimization procedures solved with evolutionary stochastic optimization algorithms. The accuracy of this method was evaluated on 57 scoliotic vertebrae in vivo. The mean difference of point to surface from 3D model from CT-scan (with the accuracy of $1 \mathrm{~mm}$ ) was $1.46 \mathrm{~mm}$ for lumbar and $1.3 \mathrm{~mm}$ for thoracic vertebrae. Their method reduced the user intervention considerably but it was used only for a short part of the spine which has less overlapping. Besides, the authors did not mention the computation time. As it is mentioned in (Moura et al, 2011), the success of these procedures for automating reconstruction based on $2 \mathrm{D} / 3 \mathrm{D}$ registration, largely depends on the initial solution.

More recent presented methods try to improve the previous methods and decrease user intervention such as (Baka et al, 2011) for distal femur. Baka et al (2011) proposed a similar method to (Benameur et al, 2003) using an automatic edge selection scheme, with different applied similarity metrics. They optimized the 3D error of the SSM, instead of a 2D error of the projected contours in (Benameur et al, 2003). They showed the accuracy of the mean RMS of $1.68 \mathrm{~mm}$ for 3D reconstruction from low quality fluoroscopic X-ray images. However, the authors reported the computation time of $5 \mathrm{~min}$ which is high in comparison to other reconstruction methods.

\subsection{Parametric Methods}

For improving the limitations of the previous methods such as time, methods are proposed based on parametric modelling and taking anatomic and clinical considerations into account. Instead of using the full set of points as proposed by SSM modelling, statistics are performed on anatomical descriptive parameters (DP) extracted from the surface of interest (S. Quijano et al, 2013). These approaches are appropriate for providing a fast and robust initial $3 \mathrm{D}$ reconstruction. These methods specify a simplified parametric model to represent the interest objects. This simplified parametric model is made of different geometric primitives such as points, lines, circles and spheres according to the considered bony structure. For instance, the parametric models used for vertebra and spine are made of points and axes (figure $4(\mathrm{~A}, \mathrm{~B})$ ). The geometric primitives are described by geometric parameters called descriptors (Lebailly et al, 2012). By applying this simplified parametric model, a database of parameters is built from the training 3D models for statistical inferences. Main descriptors are calculated according to anatomical landmarks digitized on calibrated radiographs. When the main descriptors are computed, the full set of DP are estimated according to the statistical model. From the full set of DP, the simplified personalized parametric model (SPPM) is obtained. The deformation of the morpho-realistic 3D generic mesh into the SPPM leads to the morpho-realistic personalized parametric model (MPPM) (S. Quijano et al, 2013). Finally, the 3D model is projected on the radiographs for possible adjustments and matching the information of the images and the model. These methods have been used to 3D reconstruct different bony structures such as vertebrae, spine, pelvis, lower limbs, upper limbs and rib cage which some of them are illustrated here. Pomero et al (2004) proposed a semi-automated method relied on parametric models of vertebrae using transversal statistical inferences. Their method used a prior knowledge of the vertebral shape applying eight morphologic descriptors of the 
vertebral body. The transversal inferences were based on the relationships between geometric descriptors of a given vertebra. In this method, only eight points per vertebra (the four corners of the vertebral body in each radiograph) were needed to be identified. Figure 4(B) shows the parametric model of this method. They applied a large database of 1628 dry normal and scoliotic vertebrae collected from direct measurements and 1574 normal and scoliotic vertebrae reconstructed from stereoradiography (Pomero et al, 2004). The time of whole reconstruction of the spine was 15-20 minute. They reported the mean error of 1.4and 2RMS of $3.6 \mathrm{~mm}$ for reconstruction. For $3 \mathrm{D}$ reconstruction of the thoracic and lumbar spine from radiographs, Humbert et al (2009) proposed a parametric method based on longitudinal and transversal inferences to reduce the needed time of the Pomero et al (2004) method. In this method, the parametric spine model is described by the length of a curve passing through the vertebral body centers (spinal curve), the depth, the width and the position along the spinal curve of each vertebral endplate. For inferring the shape of the spine, multi-linear regression was applied. They proposed two reconstruction levels: (a) a fast estimate of the 3D reconstruction and accurate clinical measurements with reconstruction time of $2.5 \mathrm{~min}$, (b) a more accurate 3D reconstruction using a fine adjustment of the $3 \mathrm{D}$ models, with the reconstruction time of $10 \mathrm{~min}$. The mean shape accuracy in comparison with CT-scan was $1.0 \mathrm{~mm}$. However, their method still require considerable user-interaction, making the reconstruction user-dependent and potentially less reproducible (Moura et al, 2011). Figure 4(A) shows the parametric spine model (for L4 and L5) of this method.

For 3D reconstruction of the proximal femur, Baudoin et al (2008) proposed a parametric algorithm. Their geometric model was based on a database of proximal epiphysis of 60 femurs database. Their simplified 3D model was made of geometric object such as sphere and truncated cone. From the X-ray exploitation and the use of a database, a simplified personalized parametric model was evaluated. A morphorealistic generic model was added from $\mathrm{CT}$ to obtain a morphorealistic personalized parametric model (figure 4C). The MPPM was calculated using multiple linear regressions. Then, the obtained morpho-realistic personalized parametric model was used as the generic model for the NSCC algorithm. The mean error obtained in comparison to CT scans was $1.0 \mathrm{~mm}$ with the maximum value of $5.1 \mathrm{~mm}$ in ideal conditions. The reproducibility of this method was acceptable for obtaining 3D reconstructions of the femur proximal epiphysis from biplanar $\mathrm{X}$-rays. The reconstruction time for the whole process was approximately $4 \mathrm{~min}$.

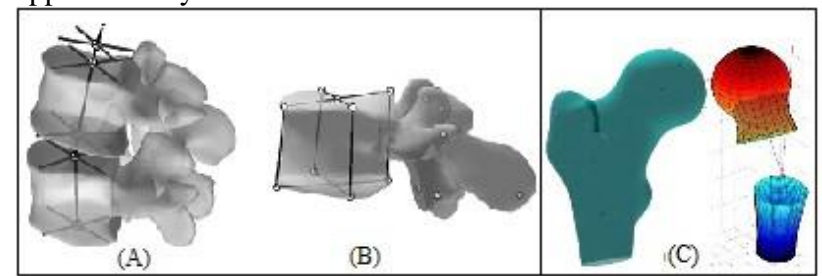

Fig. 4.(A) Parametric spine model (S. Humbert et al, 2009), (B) Parametric vertebra model for L4, (C) (left) MPPM, (right) SPPM for the proximal femur (A. Baudoin et al, 2008).

Chaibi et al (2011) proposed a method based on parametric models and statistical inferences. Their reconstruction method was performed in two steps. The first step was fast 3D lower limb reconstruction. In this step, a SPPM was defined by representing main features of each bone with geometrical primitives such as points, spheres, cylinders and segments. The
SPPM was generated by multi linear regression. The second step is full 3D lower limb reconstruction. Using the SPPM position and geometry, the MPPM was built and projected on both radiographs. A fine adjustment of anatomical landmarks and contours was done, providing a more detailed model. After the full reconstruction, additional clinical measurements, such as femoral mechanical angle were calculated. Their parametric method focused more on the femur. For the femur, the shape accuracy in comparison with the CT-scan for 11 cadaveric femurs showed mean difference of $1.0 \mathrm{~mm}(\max : 6.6)$ with 2 RMS of $2.4 \mathrm{~mm}$ which are similar to results obtained by (Laporte et al. 2003) and (Baudoin et al. 2008). The mean time for reconstruction was about $10 \mathrm{~min}$ for both lower limbs. It showed that, this method can reach to the acceptable accuracy in shorter time with reduced digitalization process.

Quijano et al (2013) improved the previous method proposed by Chaibi et al (2011) by using a new parametric model of the tibia and a different regression approach. They applied a CTscan morphometric database of femurs and tibias. This CT based database consisted of 56 femurs and 24 tibias. A set of anatomical landmarks was digitized, and employed to calculate the main descriptors of the statistical model. Main descriptors used for the femur such as head ratio, lateral and medial condyle radius, and main descriptors for the tibia such as length, were calculated. The full set of DP was obtained via statistical inferences of the main descriptors. The improvement was related to the accuracy of the descriptors calculated via partial least squares regressions instead of multi linear regression which is a classic regression technique applied in previous method. They showed that the improvement of the 'fast 3D' reconstruction permits skipping the manual adjustments required. Comparison to the previous method showed an improvement of the Initial Solution in terms of shape accuracy. The 3D reconstructions from CT-scan were used as references for accuracy assessment. For the femur, the mean (2RMS) shape error was $1.3 \mathrm{~mm}(3.5 \mathrm{~mm})$, less than the $1.6 \mathrm{~mm}(4.2 \mathrm{~mm})$ of Chaibi et al (2011) for the 'fast 3D' reconstruction. For the tibia, the results were $1.3 \mathrm{~mm}(3.2 \mathrm{~mm})$, instead of $2 \mathrm{~mm}(5 \mathrm{~mm})$ and $1.2 \mathrm{~mm}(3.2 \mathrm{~mm})$ obtained respectively with the fast and 'full 3D-I' of the previous method for 9 tibias (Quijano et al, 2013). The fast 3D reconstruction method of Quijano et al (2013) needs time of (3-4.6 min) which is more than the previous method.

In addition, Lebailly et al, (2012) and Aubert et al, (2014) also proposed similar parametric methods for $3 \mathrm{D}$ reconstruction from biplanar images of upper limb and rib cage, respectively, with respect to their application. The results and characteristics of their 3D reconstruction algorithms are shown in table1, briefly.

\subsection{Hybrid Methods}

This class belongs to methods obtained from the integration of different types of models from various sources. Although these methods may have similar characteristics with the previous mentioned method classes, they can be considered as a distinct class called hybrid methods due to their characteristics. Methods of this class can have different properties with regards to their combinations and applications. A hybrid method would be illustrated in the following. Kadoury et al (2009, 2015) offered a hybrid statistical and image-based biplanar reconstruction method for the spine. Their hybrid 3D reconstruction method merged statistical knowledge with image based information. In their approach, the spine centerlines extracted from the pre-operative images are used to map the 3D reconstruction of the spinal curve in a low-dimensional representation of a scoliotic database, and perform a statistical 
modeling of the anatomy based on an analytical regression (Kadoury, 2015). Local linear embedding (LLE) was used for mapping 3D splines to a lower dimensional space, which was then used to infer the spine reconstruction using support vector regression (SVR). The model is refined locally at each vertebral level via a segmentation method based on a level set surface evolution paradigm. Their method requires computation time of $2.4 \mathrm{~min}$ in addition to the time needed for identifying the splines for 3D reconstruction of the spine. This method uses a large database for creating the statistical model. This was needed since the statistical approach proposed by the authors is based on local linear embedding (LLE) and this technique is sensible to insufficient sampling (Moura, et al, 2011). The mean point-to-surface errors between the reconstructed 3D vertebral models from the proposed method and MRI are $1.2 \pm 1.1 \mathrm{~mm}$ for lumbar vertebra and $1.1 \pm 0.8 \mathrm{~mm}$ for thoracic vertebra. This method has acceptable accuracy for patients with moderate scoliosis.

\section{DISCUSSION AND CONCLUSION}

In this section, the mentioned methods are evaluated and their advantages and disadvantages are described. In addition, a comparison has been done on the presented methods.

Point-based methods are heavily dependent on the skill of the operator. It is difficult to accurately identify and match points on multi-view radiographs. Therefore, point-based methods cannot ensure reproducibility. Besides, when SCP-based methods are applied, because of the limited number of corresponding anatomical landmarks identifiable on radiographs, these methods have limited accuracy. Point-based methods cannot be used for bony structures with continuous shape because of the lack of specific anatomical landmark points. Moreover, manual identification of landmarks is time consuming and complex. Due to the required manual identification, the time of reconstruction for these methods is about 2-4 hours. Therefore, these methods are time consuming. This would be a serious disadvantage which cannot be neglected especially for clinical application. Despite of the mentioned disadvantages such as low reproducibility and time consuming, point based methods still are applied for 3D reconstruction because of simplicity of their processes (not for the operator). These methods are usually used for presenting an initial solution for other methods. In contour based methods, because of using contours instead of points, user intervention is reduced in comparison to point based methods. The time of 3D reconstruction for these methods is about 15-35 minute for lower limbs which is less than point-based methods. However, these methods are still considered time consuming for being applied in clinical applications. The accuracy of these methods is acceptable and close to CT scan results. In contour based methods, the problem of finding corresponding points is avoided and it reduces the need for user intervention in comparison to point-based methods. The statistical shape model based methods are more automated with respect to the previous mentioned algorithms. They reduce user intervention with considering more information about pathologic objects. Besides, they achieve accurate 3D reconstruction especially for objects such as vertebrae. However, these methods need a large learning database. The total reconstruction time has not been reported precisely. Parametric approaches are appropriate for providing a fast and robust initial $3 \mathrm{D}$ reconstruction. They improved the robustness and convergence of the algorithms. The results of these algorithms are enhanced with acceptable reproducibility especially for lower limb bony structures. However, for using this type of methods, the application must be considered. It should be mentioned that parametric model based methods can reach to the acceptable accuracy in shorter time with reduced digitalization process particularly for lower limb. Hybrid methods can have different properties with regards to their combinations. They are applied by considering the requirements. These methods can improve the results if they are consistent to the application. However, they have limitations due to their combinations of methods. A sample hybrid method illustrated in this paper was proposed by Kadoury et al. $(2009,2015)$ for 3D reconstruction of spine. This method used a large database but it has acceptable accuracy for patients with moderate scoliosis. However, only the computation time was reported for their reconstruction algorithm. Therefore, an appropriate evaluation of time cannot be done for their method.

With regards to the mentioned characteristics, each proposed method in this study has several advantages and disadvantages which should be considered for the usage, and no method can be chosen as the best for all applications. Table 1 presents several 3D reconstruction methods for different applications from mentioned classes. Their accuracy, time, validation reference and imaging systems of methods in different studies for 3D reconstruction from X-ray images are mentioned for various bony structures. It should be considered that some of the presented methods used EOS system, instead of standard radiographic systems for image capturing. EOS system captures simultaneous X-ray images with higher quality. This affects the accuracy and the time of the reconstruction methods. Better time can be achieved if EOS systems are applied. In table 1 imaging systems of presented methods (EOS system or standard radiographic systems) are indicated for the comparison. 
Table1: Evaluation of 3D Reconstruction methods from $\mathrm{X}$ ray images

\begin{tabular}{|c|c|c|c|c|c|}
\hline $\begin{array}{l}\text { Authors } \\
\text { (date) }\end{array}$ & $\begin{array}{c}\mathrm{X} \text { ray } \\
\text { imaging }\end{array}$ & $\begin{array}{l}\text { Reconstruction } \\
\text { method }\end{array}$ & $\begin{array}{c}\text { Validatio } \\
n \\
\text { reference }\end{array}$ & Time $(\mathrm{h}, \mathrm{m}, \mathrm{s})$ & Errors(mm) \\
\hline \multicolumn{6}{|l|}{ Pelvis: } \\
\hline $\begin{array}{c}\text { Gauvin et al } \\
(1998)\end{array}$ & $\mathrm{SRS}^{1}$ & $\mathrm{SCP}$ & $\mathrm{DM}^{2}$ & & $2 \mathrm{SD}=4.8$ \\
\hline $\begin{array}{c}\text { Mitton et al } \\
(2006)\end{array}$ & EOS & NSCC & СT & $15-35 \mathrm{~m}$ & mean $=1.6,2 \mathrm{RMS}=4.3$ \\
\hline \multicolumn{6}{|c|}{ Vertebrae, Spine: } \\
\hline $\begin{array}{c}\text { Aubin et al } \\
\text { (1997) }\end{array}$ & $\mathrm{SRS}^{1}$ & $\mathrm{SCP}$ & $\mathrm{DM}^{2}$ & \multirow{4}{*}{ about $2-4 \mathrm{~h}$} & $6 \mathrm{SCP}:$ mean=2.6, $21 \mathrm{SCP}:$ mean $=2.1$ \\
\hline $\begin{array}{l}\text { Mitton et al } \\
(2000)\end{array}$ & $\mathrm{SRS}^{1}$ & NSCP & $\mathrm{DM}^{2}$ & & mean= about 1 \\
\hline $\begin{array}{c}\text { Mitulescu et } \\
\text { al (2001) }\end{array}$ & $\mathrm{SRS}^{1}$ & NSCP & $\mathrm{DM}^{2}$ & & mean $=1.1,2 \mathrm{RMS}=2.8$ \\
\hline $\begin{array}{c}\begin{array}{c}\text { Mitulescu et } \\
\text { al (2002) }\end{array} \\
\end{array}$ & $\mathrm{SRS}^{1}$ & NSCP & CT & & mean $=1.5,2 \mathrm{RMS}=4.0$ \\
\hline $\begin{array}{c}\text { Benameur et } \\
\text { al (2003) }\end{array}$ & $\mathrm{SRS}^{1}$ & SSM & СT & & $\begin{array}{l}\text { lumbar vertebra: } \text { mean }=0.71 \text {, } \\
\text { thoracic vertebra: } \text { mean }=1.48\end{array}$ \\
\hline $\begin{array}{c}\text { Benameur et } \\
\text { al (2005) }\end{array}$ & $\mathrm{SRS}^{1}$ & SSM & CT & & $\begin{array}{l}\text { lumbar vertebra: mean }=1.46 \text {, } \\
\text { thoracic vertebra: } \text { mean }=1.3\end{array}$ \\
\hline $\begin{array}{c}\begin{array}{c}\text { Pomero et al } \\
\text { (2004) }\end{array} \\
\end{array}$ & $\mathrm{SRS}^{1}$ & Parametric & CT & $\begin{array}{l}\text { fast: } 14 \mathrm{~m}, \\
\text { refined:20m }\end{array}$ & mean=1.4, 2RMS=3.6 \\
\hline $\begin{array}{c}\text { Humbert et al } \\
(2009)\end{array}$ & EOS & Parametric & CT & $\begin{array}{l}\text { fast: } 2.5 \mathrm{~m}, \\
\text { refined: } 10 \mathrm{~m}\end{array}$ & $\begin{array}{c}\text { fast: mean=1.3, } 2 \mathrm{RMS}=3.6, \\
\text { refined: } \text { mean=1.0, } 2 \mathrm{RMS}=2.7\end{array}$ \\
\hline \begin{tabular}{c|} 
Kadoury et \\
al(2009)
\end{tabular} & $\mathrm{SRS}^{1}$ & Hybrid & MRI & Comp ${ }^{3}$ time: $2.4 \mathrm{~m}$ & $\begin{array}{l}\text { lumbar vertebra: mean }=1.2, \\
\text { thoracic vertebra: } \text { mean=1.1 }\end{array}$ \\
\hline \multicolumn{6}{|c|}{ Lower Limb: Femur, Tibia, ,... } \\
\hline $\begin{array}{c}\begin{array}{c}\text { Fleute et al } \\
(1999)\end{array} \\
\end{array}$ & $\mathrm{SRS}^{1}$ & SSM & CT & Compstime: $1 \mathrm{~m}$ & Distal Femur: RMS=1 \\
\hline $\begin{array}{c}\text { Laporte et al } \\
\text { (2003) }\end{array}$ & $\mathrm{SRS}^{1}$ & NSCC & CT & $15 \sim 35 \mathrm{~m}$ & $\begin{array}{l}\text { Distal Femur: mean }=1,2 \mathrm{RMS}=2.8 \text {, } \\
\qquad \max =5\end{array}$ \\
\hline $\begin{array}{c}\text { Baudoin et al } \\
(2008)\end{array}$ & EOS & Parametric & CT & $\sim 4 \mathrm{~m}$ & Proximal Femur: $\operatorname{mean}=1, \max =5.1$ \\
\hline $\begin{array}{c}\begin{array}{c}\text { Baka et al } \\
(2011)\end{array} \\
\end{array}$ & $\mathrm{SRS}^{1}$ & SSM & CT & $5 \mathrm{~m}$ & Distal Femur: RMS=1.6mm \\
\hline $\begin{array}{c}\text { Chaibi et al } \\
\text { (2011) }\end{array}$ & EOS & Parametric & СT & $\begin{array}{l}\text { fast: } 2 \mathrm{~m}, \\
\text { full: } 10 \mathrm{~m} \text { for both } \\
\quad \text { limb }\end{array}$ & $\begin{array}{c}\text { Femur:(fast):mean=1.6,2RMS }=4.2 \\
\text { (full):mean }=1,2 \mathrm{RMS}=2.4 \text {, Tibia: } \\
\text { (fast):mean }=2,2 \mathrm{RMS}=5, \\
\text { (full):mean }=1,2 \mathrm{RMS}=3.2\end{array}$ \\
\hline $\begin{array}{c}\text { Quijano et } \\
\text { al (2013) } \\
\end{array}$ & EOS & Parametric & CT & fast: $3 \sim 4.6 \mathrm{~m}$ & $\begin{array}{c}\text { Femur:(fast):mean }=1.3,2 \mathrm{RMS}=3.5, \\
\text { Tibia:(fast):mean=1.3, 2RMS=3.2 }\end{array}$ \\
\hline \multicolumn{6}{|l|}{ Upper Limb: } \\
\hline $\begin{array}{l}\text { Lebailly et al } \\
\text { (2012) }\end{array}$ & EOS & Parametric & CT & $5 \mathrm{~m}$ & mean $=0.9,2 \mathrm{RMS}=2.5, \max =6.7$ \\
\hline \multicolumn{6}{|l|}{ Rib cage: } \\
\hline $\begin{array}{c}\text { Aubert et al } \\
\text { (2014) }\end{array}$ & EOS & Parametric & CT & $6 \mathrm{~m}$ & mean $=3.6,2 \mathrm{RMS}=8.8, \max =24.3$ \\
\hline
\end{tabular}

\section{REFERENCES}

Aubin CE, Dansereau J, Parent F, Labelle H, de Guise JA. 1997. Morphometric evaluations of personalised 3D reconstructions and geometric models of the human spine. Med Biol Eng Comput. 35(6):611-618.

Aubert B, Vergari C, Ilharreborde B, Courvoisier A, Skalli, W. 2014. 3D Reconstruction of Rib Cage Geometry from Biplanar Radiographs using a Statistical Parametric Model approach

Baudoin A, Skalli W, de Guise JA, Mitton D. 2008. Parametric subject-specific model for in vivo 3D reconstruction using biplanar X-rays: application to the upper femoral extremity. Med Biol Eng Comput. 46(8):799-805.

Bauer T. 2002. Reconstruction tridimensionnelle de l'articulation du genou par stereoradiographie:De l'apprentissage in vitro a l'application clinique dans le cas de la gonarthrose [MasterThesis]. [Paris (FR)]: Laboratoire de biomecanique. Ecole Nationale Superieure d'Arts etMetiers.

BakaN, Kaptein B.L, de BruijneM, van WalsumT, GiphartJ.E, NiessenW.J, LelieveldtB.P.F. 2011.2D-3D shape reconstruction of the distal femur from stereo X-ray imaging usingstatistical shape models. Med. Image Anal. 15(6), 840-850.

Benameur S, Mignotte M, Parent S, Labelle H., Skalli W, Guise J.D. 2003. 3D/2Dregistration and segmentation of scoliotic vertebrae using statistical models.Computerized Medical Imaging and Graphics 27, 321-337.
Benameur S, Mignotte M, Labelle H, De Guise JA. 2005. A hierarchical statistical modeling approachfor the unsupervised 3 -D biplanar reconstruction of the scoliotic spine. IEEE Trans BiomedEng. 52(12):2041-2057.

Chaibi Y. Adaptation des méthodes de reconstruction 3D rapides par stéréora-diographie: Modélisation du membre inférieur et calcul des indices cliniquesen présence de déformation structurale. Paris: Arts et métiers Paristech; 2010[Thesis].

Chaibi Y, Cresson T, Aubert B, Hausselle J, Neyret P, Hauger O. 2011. Fast 3D reconstruction of the lower limb using a parametric modeland statistical inferences and clinical measurements calculation frombiplanar X-rays. Comput Methods Biomech Biomed Eng 2011.

Fleute M. 2001. Shape Reconstruction for Computer Assisted Surgery based on Non-Rigid Registration of Statistical Models with Intra-Operative Point Data and X-ray Images [Ph.D thesis], Universite Joseph Fourrier.

Humbert L, De Guise JA, Aubert B, Godbout B, Skalli W. 2009.3D reconstruction of the spine from biplanar X-rays using parametric models based on transversal and longitudinal inferences. Med Eng Phys. 31(6):681-687.

Hurvitz, A., Joskowicz, L., 2008. Registration of a ct-like atlas to fluoroscopic X-ray images using intensity correspondences. International Journal of Computer Assisted Radiology and Surgery (CARS) 3, 493-504.

Kadoury S, Cheriet F, Labelle H., 2009, Personalized X-ray 3$\mathrm{D}$ reconstruction of the scoliotic spine from hybrid statistical and image-based models. IEEE Trans Med Imaging 2009;

Kadoury S.2015. Three-Dimensional Spine Reconstruction from Radiographs., Springer International Publishing Switzerland, Spinal Imaging and Image Analysis. Lecture Notes in Computational Vision and Biomechanics 18.

Laporte S. 2002. Reconstruction 3D du squelette humain pour la biomecanique par radiographie biplane a dose minimale d'irradiation [Paris]: Laboratoire de Biomecanique. Ecole Nationale Superieure d'Arts et Metiers.

Laporte S, Skalli W, de Guise JA, Lavaste F, Mitton D. 2003. A biplanar reconstruction method based on $2 \mathrm{D}$ and $3 \mathrm{D}$ contours: application to the distal femur. Comput Methods Biomech Biomed Engin. 6(1):1-6.

Le Bras A, Laporte S, Bousson V, Mitton D, De Guise JA, Laredo JD, Skalli W. 2004. 3D reconstruction of the proximal femur with low-dose digital stereoradiography. Comput Aided Surg. 9(3):51-57.

Lebailly F, Lima L. V. P. C, Clairemidi A, Aubert B, Guerard S, Chaibi Y, de Guise J, Fontaine C, Skalli W. 2012. Semiautomated stereoradiographic upper limb 3D reconstructions using a combined parametric and statistical model: a preliminary study,Surg Radiol Anat (2012) 34:757-765.

Mahfouz M, Badawi A, Abdel Fatah E, Kuhn M, Merkl B. 2006. Reconstruction of 3D Patient-Specific Bone Models From Biplanar X-ray Images Utilizing Morphometric Measurements. World Congress in Computer Science Computer Engineering and Applied Computing. Las Vegas, Nevada. 
Markelj, P, Tomaževič, D, Likar, B, Pernuš, F. 2010. A review of $3 \mathrm{D} / 2 \mathrm{D}$ registration methods for image-guided interventions. Medical Image Analysis.

Mitton D, Landry C, Veron S, Skalli W, Lavaste F, De Guise JA. 2000. 3D reconstruction method from biplanar radiography using non-stereo corresponding points and elastic deformable meshes. Med Biol Eng Comput. 38(2):133-139.

Mitton D, Deschenes S, Laporte S, Godbout B, Bertrand S, de Guise JA, Skalli W. 2006. 3D reconstruction of the pelvis from bi-planar radiography. Comput Methods Biomech Biomed Engin. 9(1):1-5.

Mitulescu A, Semaan I, De Guise JA, Leborgne P, Adamsbaum C, Skalli W. 2001. Validation of the non stereo corresponding points stereo radiographic $3 \mathrm{D}$ reconstruction technique. Med Biol Eng Comput. 39(2):152-158.

Mitulescu A, De Guise JA, Dubousset J, Labelle H, Adamsbaum C, Skalli W. 2002. Validation of the NSCP technique on scoliotic vertebrae. Stud Health Technol Inform. 88:167-171.

Mitulescu A, Laporte S, Boulay C, De Guise JA, Skalli W. 2002. 3D reconstruction of the pelvis using the NSCP technique. Stud Health Technol Inform. 88:177-181.

Moura DC, Boisvert J, Barbosa JG, Labelle H, Tavares JM. 2011. Fast3D reconstruction of the spine from biplanar radiographs using deformable articulated model. Med Eng Phys 2011.

Pearcy MJ. 1985. Stereo radiography of lumbar spine motion. Acta Orthop Scand Suppl. 212:1-45.

Pomero V, Mitton D, Laporte S, de Guise JA, Skalli W. 2004. Fast accurate stereoradiographic 3Dreconstruction of the spine using a combined geometric and statistic model. Clin Biomech (Bristol, Avon). 19(3):240-247.

Quijano S., Serrurier A., Aubert B., Laporte S., Thoreux P., Skalli W. 2013. Three-dimensional reconstruction of the lower limb from biplanar calibrated radiographs. Med. Eng. Phys. 35 (12), 1703-1712.

Sadowsky O, Chintalapani G, Taylor RH. Deformable 2D-3D registration of the pelvis with a limited field of view, using shape statistics. Med Image Com-put Comput Assist Interv Int Conf Med Image Comput Comput Assist Interv2007;10:519 26.

Tang T, Ellis R. 2005. 2D/3D deformable registration using a hybrid atlas. Medical Image Computing and Computer-Assisted Intervention 8, 223-230.

Yao J, Taylor R. 2003. Assessing accuracy factors in deformable 2D/3D medical image registration using a statistical pelvis model. In: ICCV '03: Proceedings of the Ninth IEEE International Conference on Computer Vision. IEEE Computer Society, Washington, DC, USA, p. 1329.

Zheng, Gollmer G, Schumann S, Dong X, Feilkas T, Ballester M.A.G. 2008. A 2D/3D correspondence building method for reconstruction of a patient-specific 3D bone surface model using point distribution models and calibrated X-ray images. Medical Image Analysis 13 (6), 883-899. 